Research Paper

\title{
Validation study of susceptibility loci for esophageal squamous cell carcinoma identified by GWAS in a Han Chinese subgroup from Eastern China
}

\author{
Kai-Lai Wang ${ }^{1 *}$, Xiang-Liu Chen ${ }^{*}$, Lan Lei ${ }^{*}$, Pei Li ${ }^{*}$, Lian-Lian Hong1, Xian-Chong Huang1, Wei-Min \\ Mao $^{3,4}$, Kenichi Mukaisho5 ${ }^{5}$ Zhi-Qiang Ling1, ${ }^{1}$ \\ 1. Zhejiang Cancer Institute, Zhejiang Cancer Hospital, No.1 Banshan East Rd., Gongshu District, Hangzhou 310022, P.R.China. \\ 2. Department of Pathophysiology, School of Basic Medical Sciences, Zhengzhou University, Zhengzhou, 450052, China. \\ 3. Department of Thoracic Tumor Surgery, Zhejiang Cancer Hospital, No.1 Banshan East Rd., Gongshu District, Hangzhou 310022, P.R.China. \\ 4. Zhejiang Key Laboratory of Diagnosis \& Treatment Technology on Thoracic Oncology (Lung and Esophagus), Hangzhou 310022, China. \\ 5. Department of Pathology, Division of Molecular Diagnostic Pathology, Shiga University of Medical Science, Otsu, Shiga, Japan. \\ *Kai-Lai Wang, Xiang-Liu Chen, Lan Lei and Pei Li wish it to be known that, in their opinion, the first four authors should be regarded as joint-first authors. \\ $\square$ Corresponding author: Zhi-Qiang Ling, Professor, MD.,PhD, Zhejiang Cancer Institute, Zhejiang Cancer Hospital, Zhejiang Cancer Center. No.1 Banshan \\ East Road, Gongshu District, Hangzhou 310022, P. R. China. Tel: +86-571-88122423; Fax: +86-571-88122423; E-mail: lingzq@zjcc.org.cn or lingzq@hotmail.com \\ (c) Ivyspring International Publisher. This is an open access article distributed under the terms of the Creative Commons Attribution (CC BY-NC) license \\ (https:// creativecommons.org/licenses/by-nc/4.0/). See http://ivyspring.com/terms for full terms and conditions.
}

Received: 2019.01.04; Accepted: 2019.05.04; Published: 2019.06.09

\begin{abstract}
Esophageal squamous cell carcinoma (ESCC) occurs at a relatively high frequency in China and is one of the most prevalent cancers in the world. Genome-wide association studies (GWAS) have identified 24 single-nucleotide polymorphisms (SNPs) that could be associated with ESCC in Chinese patients. This retrospective study aimed to validate the association between these 24 SNPs and ESCC in a Han Chinese subgroup from East China. A total of 2280 and 1900 patients with ESCC (case group) and non-esophageal cancer (control group) were included from a single center. Genotyping of the 24 polymorphisms was performed using the Sequenom MassARRAY system. Unconditional logistic regression analyses were conducted for every polymorphism. It was found that $\mathrm{rs} 12188136(\mathrm{P}=0.027, \mathrm{OR}=1.158,95 \% \mathrm{Cl}=1.016-1.319$ for $\mathrm{AG} / \mathrm{AA})$ was associated with $\mathrm{ESCC}$. Binary logistic regression analyses revealed a significant negative association of rs875339 in RORA $(\mathrm{P}=0.014, \mathrm{OR}=0.762,95 \% \mathrm{Cl}=0.613-0.947$ for $\mathrm{TT} / \mathrm{CC})$. Under the dominant model, rs 6854472 was slightly associated with $E S C C$ risk $(P=0.048, O R=1.192,95 \% C l=1.002-1.418)$. Under the recessive model, a significant negative association was observed for $r 5875339$ ( $P=0.010, O R=0.758,95 \%$ $\mathrm{Cl}=0.615-0.935)$. In a word, this large-scale replication study validated that $\mathrm{rs} 12188136$ and rs6854472 are associated with ESCC in a Han Chinese subgroup from Eastern China, and that rs875339 is negative associated with ESCC.
\end{abstract}

Key words: esophagi al squamous cell carcinoma (ESCC), genome-wide association study (GWAS), single nucleotide polymorphism (SNP), MassARRAY system, Han Chinese population

\section{Introduction}

Esophageal cancer is among the most incident malignant tumors worldwide [1] and a serious threat to human health and quality of life [2]. Esophageal squamous cell carcinoma (ESCC) is one of the two main sub-types of esophageal cancer, and ESCC is more common than esophageal adenocarcinoma in the developing world, especially in China [3]. The prognosis of ESCC is poor despite advances in treatment, with 5-year overall survival rate ranging from $15 \%$ to $25 \%[4,5]$.

Accumulating evidence has demonstrated that genetic factors [6-10], family history of ESCC [11-13], lifestyle habits [14-16], environmental factors [17-23], and HPV infection [24] play important roles in the 
development of ESCC. Significant interactions were found between HPV serological status and genetic loci, increasing the risk of ESCC $[25,26]$. Other risk factors such as exposure to polycyclic aromatic hydrocarbons (PAHs), high-temperature foods, diets, oral health and microbial communities, but they require further research. Esophageal carcinogenesis is the result of the interaction among heredity, environment and living habits [27-29].

In recent years, genome-wide association studies (GWAS) have confirmed the contribution of gene variations to ESCC [30-35]. Six large-scale GWAS of Chinese populations have focused on identifying genetic susceptibility loci for ESCC [31-35]. The earliest ESCC GWAS analysis using 2115 ESCC cases and 3302 controls in a Chinese population revealed that PLCE1 carried cancer susceptibility [31]. Wang et al. identified two new genome-wide significant loci for ESCC: PLCE1 at 10q23 and C20orf54 at 20p13 [32]. Seven loci on chromosomes 5q11, 6p21, 10q23, 12q24 and 21q22 were associated with the risk of ESCC [33]. In another GWAS in a Chinese ESCC population, $\mathrm{Wu}$ et al. [34] identified nine new ESCC susceptibility loci: seven (on chromosomes 4q23, 16q12.1, 17q21, 22q12, $3 \mathrm{q} 27,17 \mathrm{p} 13$ and 18p11) had a significant marginal effect on the risk of ESCC and two (on 2q22 and 13q33) had a significant association but only when considering the gene-alcohol interaction. Wu et al. identified rs1050631 in SLC39A6 as being associated with the survival of ESCC patients [35].

Whether those 24 SNPs found by the five GWAS confer an increased risk of ESCC in various Han Chinese populations has not yet been validated. Therefore, we conducted a case-control study to validate the associations of those 24 SNPs with the risk of ESCC in a Han Chinese subgroup from Eastern China.

\section{Material and Methods}

\section{Study population}

This was a retrospective study. We included 2280 consecutive ESCC subjects and 1900 non-ESCC subjects (control group). The diagnosis of ESCC was confirmed by histopathology or cytology by at least two local pathologists. Histological examination was performed according to the World Health Organization (WHO) criteria [36]. The exclusion criteria for both groups were: 1) psychiatric disorder; 2) any other primary cancer; or 3) a family history of cancer. This study consisted of two ESCC sets: (a) 1900 patients with primary ESCC, and (b) 380 patients with second ESCC. The patients were recruited between January 2012 and December 2014 at Zhejiang Cancer Hospital. Demographic characteristics of the subjects (including gender, age, histological types of esophageal cancer, smoking and drinking status) were obtained from the medical records. Non-ESCC individuals $(n=1900)$ were recruited as control subjects during a routine health check-up (physical examination) at the same hospital during the same time period. The two groups were matched based on the frequency of age and sex. In the present study, all participants were ethnic Han Chinese that lived within the Zhejiang Province of Eastern China.

\section{SNP selection}

We selected the 24 top SNPs (rs4478858, rs10881372, rs10801638, rs10173378, rs888103, rs38155 01, rs6717108, rs10934685, rs6768588, rs9824873, rs685 4472, rs12188136, rs2294693, rs9364414, rs7916519, rs11225815, rs10895458, rs4578395, rs11059556, rs2025 245, rs9584006, rs347940, rs875339, and rs12922317) from the reports focusing on ESCC susceptibility loci identified by five GWAS projects in Han Chinese (PubMed search) [31-35].

\section{SNP genotyping assays}

Venous blood $(2 \mathrm{~mL})$ was sampled in citrate glass tubes and kept at $-40^{\circ} \mathrm{C}$. Leukocyte total genomic DNA was extracted from $1 \mathrm{~mL}$ of peripheral blood using the Whole Blood DNA Extraction Kit (QIAamp ${ }^{\circledR}$ DNA Blood Mini Kit), according to the manufacturer's instructions. The extracted genomic DNA was dissolved in $0.1 \times$ TE buffer $(10$ mMTris and $1 \mathrm{mM}$ EDTA, $\mathrm{pH} 8.0$ ) to $0.4-0.6 \mathrm{mg} / \mathrm{mL}$ and stored at $-20^{\circ} \mathrm{C}$.

The SNPs were determined using iPLEX chemistry on a matrix-assisted laser desorption/ ionization time-of-flight mass spectrometer (MALDITOF-MS, MassARRAY system, Sequenom, Inc.), as previously published [37]. PCR reactions ( $5 \mu \mathrm{L}$ each) were carried out in 384-well plates using $10 \mathrm{ng}$ of genomic DNA, 0.5 units of Taq polymerase (HotStarTaq, Qiagen), $500 \mu \mathrm{mol}$ of each of the four deoxynucleotides triphosphate (dNTP), and $100 \mathrm{nmol}$ of each primer. An ABI-9700 thermocycler was used with the following program: 1) $15 \mathrm{~min}$ at $94^{\circ} \mathrm{C}$; 2) 45 cycles of $20 \mathrm{~s}$ at $94^{\circ} \mathrm{C}, 30 \mathrm{~s}$ at $56^{\circ} \mathrm{C}$, and $60 \mathrm{~s}$ at $72^{\circ} \mathrm{C}$. The reaction products were separated on $2.0 \%$ agarose. After PCR, 0.3 units of shrimp alkaline phosphatase was added and incubated at $37^{\circ} \mathrm{C}$ for 20 min followed by inactivation for $5 \mathrm{~min}$ at $85^{\circ} \mathrm{C}$. The concentration of the extension primers was adjusted to optimize the signal-to-noise ratio. The iPLEX Gold Kits (Sequenom, Inc.) was used to prepare the samples with $0.2 \mu \mathrm{L}(100 \mu \mathrm{mol})$ of termination mix, 0.05 units of DNA polymerase (Sequenom, Inc.), and 625 to $1250 \mathrm{nmol} / \mathrm{L}$ extension primers. The iPLEX reaction was performed using the following program: 
1) initial denaturation for $30 \mathrm{~s}$ at $\left.94^{\circ} \mathrm{C} ; 2\right) 5 \mathrm{~s}$ at $94^{\circ} \mathrm{C}$ and five cycles of $5 \mathrm{~s}$ at $52^{\circ} \mathrm{C}$ and $5 \mathrm{~s}$ at $80^{\circ} \mathrm{C}$; 3) 40 annealing and extension cycles; 4) $5 \mathrm{~s}$ at $94^{\circ} \mathrm{C}$; 5) five cycles of $5 \mathrm{~s}$ at $52^{\circ} \mathrm{C}$ and $5 \mathrm{~s}$ at $80^{\circ} \mathrm{C}$; and 6$) 72^{\circ} \mathrm{C}$ for 3 min and the sample The products were analyzed by MALDI-TOF-MS. The samples were desalted using 6 $\mathrm{mg}$ of resin and transferred to a 384-well SpectroCHIP (Sequenom, Inc.). The mass spectra were acquired and analyzed using the MassARRAYTyper 4.0 Software (Sequenom, Inc.). Controls were performed without template DNA. All laboratory technicians were unaware of patient status.

\section{Statistical analyses}

Values were expressed as means \pm standard deviation (SD) or numbers. Continuous variables were analyzed using the unpaired Student's $t$-test. Differences in frequencies of the alleles and genotypes between case group and control group were evaluated using the $\chi^{2}$-test. Genotype distribution and allele frequencies were compared using the chi-square test. The chi-square test was also used to examine the Hardy-Weinberg Equilibrium (HWE) in the control group ( $P$-value of $<0.05$ was considered to be statistically significant). Akaike's information criteria were used to select the most parsimonious genetic model for each SNP [38]. Odds ratios (ORs) and 95\% confidence intervals (CIs) were calculated by unconditional logistic regression analysis. All analyses were conducted with Stata statistical package (version 10.0; Stata Corp LP, College Station, TX, USA). The $P$ value of allele difference was conducted with chi-square test between esophageal cancer and control group. $P$-value $<0.05$ was considered statistically significant.

\section{Results}

\section{Characteristics of the subjects}

The demographic characteristics of the subjects are shown in Table 1 . There were no differences in age $(57.0 \pm 8.8$ vs. $56.4 \pm 9.3$ years) or gender (male, $63.1 \%$ vs. $64.5 \%$ ) between the two groups (both $P>0.05$ ).

Table 1. Demographic characteristics of ESCC cases and controls used in the study

\begin{tabular}{llll}
\hline Study & N & Age, mean (s.d.) & Sex, male (\%) \\
\hline Cases & 2280 & $57.0(8.8)$ & 64.6 \\
First ESCC & 1900 & $57.0(9.4)$ & 64.5 \\
Second ESCC & 380 & $56.8(9.0)$ & 64.7 \\
Controls & 1900 & $56.4(9.3)$ & 64.5 \\
\hline
\end{tabular}

\section{Individual SNP association analysis}

The genomic characteristics of 24 SNPs are given in Table 2. There was no deviation from the
Hardy-Weinberg equilibrium in the control group (all $P>0.01)$. In the single-locus analyses, the allelic frequencies of rs10173378: $\mathrm{A}>\mathrm{G}(0.241$ vs. $0.221, P=$ $0.0409)$ and rs6854472: $\mathrm{G}>\mathrm{T}(0.072$ vs. $0.084, P=$ 0.0477 ) were slightly different between the ESCC and control group, but 100,000 permutations showed that there were no significant differences between the two groups. The genotype distributions of the 24 SNPs in the two groups are summarized in Table 3. The distribution of the rs12188136 $(47.4 \%$ vs. $50.2 \%, P=$ $0.0493)$ and rs875339 (49.4\% vs. $48.4 \%, P=0.0341)$ genotypes showed significant differences between the cases and controls.

Logistic regression analyses revealed that in the codominant-effect model, the ESCC risk was associated with $\mathrm{rs} 12188136(P=0.027, \mathrm{OR}=1.158,95 \% \mathrm{CI}$ $=1.016-1.319$ for AG/AA). Binary logistic regression analyses revealed a slight negative association of rs10895458 $(P=0.044, \mathrm{OR}=0.547,95 \% \mathrm{CI}=0.304-$ 0.983 for CC/AA) and a significant negative association of $\operatorname{rs} 875339(P=0.014, \mathrm{OR}=0.762,95 \% \mathrm{CI}=$ 0.613-0.947 for TT/CC), but because of the rarity of the homozygous mutant genotype $(<3 \%)$, the results were invalid for rs10895458. In addition, marginal esophageal cancer risk was found for rs6854472 $(P=$ $0.056, \mathrm{OR}=1.187,95 \% \mathrm{CI}=0.995-1.417$ for $\mathrm{GT} / \mathrm{GG})$ (Table 3).

Using the dominant model, significant ESCC risk was observed for rs6854472 $(P=0.048, \mathrm{OR}=1.192$, $95 \% \mathrm{CI}=1.002-1.418)$. Using the recessive model, a significant negative association was observed for $\operatorname{rs875339}(P=0.010, \mathrm{OR}=0.758,95 \% \mathrm{CI}=0.615-0.935)$ (Table 4).

\section{Discussion}

ESCC is one of the most prevalent cancers worldwide and occurs at a relatively high frequency in China. Some recent genome-wide association studies have identified 24 single-nucleotide polymorphisms that may be associated with ESCC. This study aimed to validate the association between these 24 polymorphisms and ESCC in a Han subgroup from Eastern China. The results suggest that rs12188136 and rs6854472 are associated with ESCC in this Han Chinese subgroup, and that rs875339 is negative associated with ESCC.

This study was a large-scale study in Han Chinese patients from Eastern China that describes the association between ESCC and 24 genome-wide SNPs. Besides rs12188136 and rs6854472 localizing in intergenic areas, RORA could play a role in the development of ESCC [31-35]. Abnet et al. [31] conducted the first large-scale genome-wide association studies for ESCC using 2115 ESCC cases and 3302 controls in Chinese, and identified PLCE1 at 
10q23 for ESCC susceptibility. Then, Wang et al. [32] performed a GWAS of ESCC by genotyping 1077 individuals with ESCC and 1733 control subjects of Han Chinese descent, and found that PLCE1 and C20orf54 play important roles for ESCC carcinogenesis. Wu et al. [33] performed a GWAS on 2031 ESCC individuals and 2044 controls of Chinese descent, and evaluated promising associations in an additional 6276 cases and 6165 controls from different areas of China. They identified five chromosomal regions (5q11, 6p21, 10q23, 12q24 and 21q22) that carried seven susceptibility loci for ESCC in the Chinese population, of which three (5q11, 6p21 and 21q22) were newly discovered [33]. Wu et al. [34] reported a multistage GWAS of ESCC in 10,123 ESCC cases and 10,664 controls. This GWAS identified nine new susceptibility loci for ESCC, of which seven (4q23, 16q12.1, 17q21, 22q12, 3q27, 17p13 and 18p11) had a significant marginal effect and two of which (2q22 and 13q33) had a significant association in the gene-alcohol interaction only [34]. Among 5337 Chinese with ESCC and 5787 controls (replication in 9654 Chinese with ESCC and 10,058 controls), Wu et al. [34] showed that rs7447927at 5q31.2 and rs1642764 at $17 \mathrm{p} 13.1$ were associated with ESCC susceptibility [34]. Furthermore, $\mathrm{Hu}$ et al. [39] showed that rs2274223 was associated with reduced PLCE1 expression and increased risk of ESCC. Another replication study by Wang et al. [40] showed that the ADH1B-ADH1C-ADH7 axis was modulated by the rs1042026, rs17033, rs1614972, rs1789903 and rs17028973 SNPs. In the present study, the identified polymorphisms matched those found by the previous studies, and included rs2294693 in 6p21.1, rs11059556 in 12q24, rs6854472 in 4q22, rs12922317 in 16p13.12, and rs9824873 in 3q28. The discrepancies among studies regarding the identified loci can be due to the genetic diversity among different regions of China and of the world. Additional studies are necessary to better understand the risk of ESCC.

Table 2. Information about 24 validated SNPs.

\begin{tabular}{|c|c|c|c|c|c|c|c|c|c|c|c|}
\hline \multirow[t]{2}{*}{ Gene: locus and OMIM No.a } & \multirow[t]{2}{*}{ SNP_ID } & \multirow{2}{*}{$\begin{array}{l}\text { Chromosome } \\
\text { No. }\end{array}$} & \multirow{2}{*}{$\begin{array}{l}\text { Chromosome } \\
\text { Position }{ }^{b}\end{array}$} & \multirow{2}{*}{$\begin{array}{l}\text { Reference } \\
\text { allele }\end{array}$} & \multirow{2}{*}{$\begin{array}{l}\text { Effect } \\
\text { allele }\end{array}$} & \multicolumn{3}{|l|}{ MAFc } & \multirow[t]{2}{*}{ Pg } & \multirow{2}{*}{$\begin{array}{l}\text { P value for } \\
H W E^{h} \text { test }\end{array}$} & \multirow{2}{*}{$\begin{array}{l}\text { Genotyping } \\
\text { call Rate }(\%)^{\mathrm{i}}\end{array}$} \\
\hline & & & & & & $\mathrm{NCBI}^{\mathrm{d}}$ & controle & $\mathrm{EC}^{\mathrm{f}}$ & & & \\
\hline $\begin{array}{l}\text { SERINC2: 1p35.1 OMIN: } \\
614549\end{array}$ & rs4478858 & 1 & 31411078 & G & A & 0.200 & 0.196 & 0.213 & 0.0592 & 0.551 & 96.75 \\
\hline $1 \mathrm{p} 13$ & rs10881372 & 1 & 106210655 & $\mathrm{C}$ & $\mathrm{T}$ & 0.163 & 0.196 & 0.187 & 0.2864 & 0.224 & 97.37 \\
\hline 1q31 & rs10801638 & 1 & 198002090 & $\mathrm{C}$ & $\mathrm{T}$ & 0.349 & 0.302 & 0.304 & 0.8751 & 0.795 & 97.13 \\
\hline $2 \mathrm{p} 22$ & rs10173378 & 2 & 43119650 & A & G & 0.198 & 0.241 & 0.221 & 0.0409 & 0.600 & 97.18 \\
\hline $\begin{array}{l}\text { LYPD6: 2q23.2 } \\
\text { OMIN: } 613359\end{array}$ & rs888103 & 2 & 149370922 & C & $\mathrm{T}$ & 0.128 & 0.115 & 0.119 & 0.6138 & 0.464 & 97.32 \\
\hline $\begin{array}{l}\text { BZW1: 2q33 } \\
\text { OMIN: N.A }\end{array}$ & rs3815501 & 2 & 200821399 & G & A & 0.488 & 0.464 & 0.467 & 0.8099 & 0.448 & 97.15 \\
\hline $2 q 36$ & rs6717108 & 2 & 224696318 & $\mathrm{C}$ & $\mathrm{T}$ & 0.444 & 0.444 & 0.449 & 0.6156 & 0.265 & 96.82 \\
\hline $\begin{array}{l}\text { UMPS: 3q21.2 } \\
\text { OMIN: } 613891\end{array}$ & rs10934685 & 3 & 124747673 & $\mathrm{C}$ & $\mathrm{T}$ & 0.389 & 0.341 & 0.335 & 0.5626 & 0.677 & 96.65 \\
\hline $\begin{array}{l}\text { ITGB5: 3q21.2 } \\
\text { OMIN: } 147561\end{array}$ & rs6768588 & 3 & 124768488 & $\mathrm{~A}$ & G & 0.244 & 0.278 & 0.282 & 0.7215 & 0.475 & 96.60 \\
\hline $3 q 28$ & rs9824873 & 3 & 183583986 & $\mathrm{~T}$ & $\mathrm{C}$ & 0.291 & 0.329 & 0.325 & 0.7073 & 0.928 & 96.17 \\
\hline $4 q 22$ & rs6854472 & 4 & 89513521 & G & $\mathrm{T}$ & 0.085 & 0.072 & 0.084 & 0.0477 & 0.871 & 97.75 \\
\hline $5 q 35$ & rs12188136 & 5 & 174407635 & A & G & 0.256 & 0.296 & 0.305 & 0.3698 & 0.222 & 96.77 \\
\hline $\begin{array}{l}\text { UNC5CL: 6p21.1 } \\
\text { OMIN: N.A }\end{array}$ & rs2294693 & 6 & 41037763 & $\mathrm{~T}$ & $\mathrm{C}$ & 0.267 & 0.253 & 0.245 & 0.4418 & 0.074 & 97.13 \\
\hline $6 \mathrm{q} 27$ & rs9364414 & 6 & 168171267 & G & A & 0.360 & 0.376 & 0.387 & 0.3088 & 0.439 & 96.79 \\
\hline $10 \mathrm{p} 12$ & rs7916519 & 10 & 23177805 & G & A & 0.140 & 0.230 & 0.234 & 0.6363 & 0.929 & 97.01 \\
\hline $\begin{array}{l}\text { DYNC2H1: 11q22.3 } \\
\text { OMIN: } 603297\end{array}$ & rs11225815 & 11 & 103469085 & $\mathrm{~T}$ & $\mathrm{C}$ & 0.233 & 0.255 & 0.248 & 0.4884 & 0.606 & 96.56 \\
\hline $11 q 22$ & rs10895458 & 11 & 103547356 & $\mathrm{~A}$ & $\mathrm{C}$ & 0.133 & 0.113 & 0.101 & 0.0938 & 0.266 & 97.30 \\
\hline $\begin{array}{l}\text { OPCML: 11q25 } \\
\text { OMIN: } 600632\end{array}$ & rs4578395 & 11 & 133242868 & $\mathrm{~T}$ & $\mathrm{C}$ & 0.105 & 0.091 & 0.088 & 0.7086 & 0.252 & 96.82 \\
\hline $12 \mathrm{q} 24.3$ & rs11059556 & 12 & 128161518 & $\mathrm{C}$ & $\mathrm{T}$ & 0.279 & 0.336 & 0.338 & 0.8632 & 0.826 & 96.41 \\
\hline $13 q 13$ & rs2025245 & 13 & 37529440 & G & A & 0.354 & 0.381 & 0.364 & 0.1169 & 0.401 & 97.22 \\
\hline $\begin{array}{l}\text { GPC5: 13q31.3 } \\
\text { OMIN: } 602446\end{array}$ & rs9584006 & 13 & 92249673 & $\mathrm{~T}$ & G & 0.372 & 0.422 & 0.406 & 0.1536 & 0.746 & 96.39 \\
\hline $\begin{array}{l}\text { FMN1: } 15 q 13.3 \\
\text { OMIN: } 136535\end{array}$ & rs347940 & 15 & 32885469 & A & G & 0.442 & 0.357 & 0.357 & 0.9613 & 0.553 & 95.96 \\
\hline $\begin{array}{l}\text { RORA: } 15 \mathrm{q} 22.2 \\
\text { OMIN: } 600825\end{array}$ & rs875339 & 15 & 60803856 & $\mathrm{C}$ & $\mathrm{T}$ & 0.314 & 0.313 & 0.295 & 0.0881 & 0.024 & 97.01 \\
\hline SNX29: 16p13.13-p13.12 & rs12922317 & 16 & 11983775 & G & A & 0.256 & 0.318 & 0.300 & 0.0916 & 0.369 & 96.82 \\
\hline
\end{tabular}

a. OMIM, Online Mendelian Inheritance in Man (http://www.ncbi.nlm.nih.gov/Omim); b. SNP position in the NCBI dbSNP Build 38 database (http://www.ncbi.nlm.nih. gov/SNP); c. MAF, minor allele frequency, representing the frequency of effect allele; $d$. MAF for Chinese in the NCBI dbSNPs database; e. MAF for control group; $f$. MAF for esophageal cancer group; g. P value, which was conducted with $x^{2}$ test, for difference in allele distributions between esophageal cancer and control group; h. HWE, Hardy-Weinberg equilibrium in control group; i. The percentage of successful genotype calls. 
Table 3. Genotype frequencies of 24 validated SNPs among cases and control and their associations with esophageal cancer risk under co-dominant genetic model.

\begin{tabular}{|c|c|c|c|c|c|c|c|c|c|c|}
\hline \multirow[t]{2}{*}{ Gene } & \multirow[t]{2}{*}{ SNP ID } & \multirow[t]{2}{*}{ Genotype } & \multicolumn{2}{|l|}{ Case } & \multicolumn{2}{|c|}{ Control } & \multirow[t]{2}{*}{$P(2 \mathrm{df})^{a}$} & \multicolumn{2}{|l|}{ Logistic regression } & \multirow[t]{2}{*}{$\mathbf{P}_{\text {trend }}$} \\
\hline & & & No. & Frequency (\%) & No. & Frequency (\%) & & OR $(95 \% \mathrm{CI})$ & $\mathbf{P b}$ & \\
\hline SERINC2 & rs4478858 & GG & 1380 & 62.22 & 1185 & 64.90 & 0.1732 & 1.000 (reference) & & 0.061 \\
\hline & & GA & 732 & 33.00 & 567 & 31.05 & & $1.109(0.969-1.268)$ & 0.133 & \\
\hline & & AA & 106 & 4.78 & 74 & 4.05 & & $1.230(0.905-1.672)$ & 0.186 & \\
\hline & rs10881372 & $\mathrm{CC}$ & 1481 & 66.00 & 1172 & 64.18 & 0.4701 & 1.000 (reference) & & 0.282 \\
\hline & & $\mathrm{CT}$ & 688 & 30.66 & 592 & 32.42 & & $0.920(0.804-1.052)$ & 0.221 & \\
\hline & & TT & 75 & 3.34 & 62 & 3.40 & & $0.957(0.678-1.352)$ & 0.804 & \\
\hline & rs10801638 & $\mathrm{CC}$ & 1096 & 49.04 & 891 & 48.82 & 0.7977 & 1.000 (reference) & & 0.876 \\
\hline & & $\mathrm{CT}$ & 920 & 41.16 & 765 & 41.92 & & $0.978(0.858-1.114)$ & 0.734 & \\
\hline & & TT & 219 & 9.80 & 169 & 9.26 & & $1.053(0.846-1.312)$ & 0.641 & \\
\hline & rs10173378 & AA & 1341 & 60.16 & 1053 & 57.45 & 0.1040 & 1.000 (reference) & & 0.039 \\
\hline & & AG & 789 & 35.40 & 678 & 36.99 & & $0.914(0.802-1.041)$ & 0.176 & \\
\hline & & GG & 99 & 4.44 & 102 & 5.56 & & $0.762(0.571-1.017)$ & 0.065 & \\
\hline LYPD6 & rs888103 & $\mathrm{CC}$ & 1743 & 77.95 & 1432 & 78.17 & 0.3918 & 1.000 (reference) & & 0.615 \\
\hline & & $\mathrm{CT}$ & 456 & 20.39 & 379 & 20.69 & & $0.988(0.848-1.152)$ & 0.882 & \\
\hline & & TT & 37 & 1.65 & 21 & 1.15 & & $1.448(0.844-2.484)$ & 0.179 & \\
\hline BZW1 & rs3815501 & GG & 633 & 28.33 & 516 & 28.24 & 0.8648 & 1.000 (reference) & & 0.809 \\
\hline & & GA & 1115 & 49.91 & 925 & 50.63 & & $0.983(0.850-1.136)$ & 0.813 & \\
\hline & & AA & 486 & 21.75 & 386 & 21.13 & & $1.026(0.860-1.225)$ & 0.773 & \\
\hline & rs6717108 & $\mathrm{CC}$ & 689 & 30.94 & 575 & 31.59 & 0.8831 & 1.000 (reference) & & 0.620 \\
\hline & & $\mathrm{CT}$ & 1075 & 48.27 & 875 & 48.08 & & $1.025(0.889-1.182)$ & 0.731 & \\
\hline & & TT & 463 & 20.79 & 370 & 20.33 & & $1.044(0.876-1.245)$ & 0.629 & \\
\hline UMPS & rs10934685 & $\mathrm{CC}$ & 972 & 43.82 & 787 & 43.19 & 0.8142 & 1.000 (reference) & & 0.560 \\
\hline & & $\mathrm{CT}$ & 1006 & 45.36 & 827 & 45.39 & & $0.985(0.864-1.123)$ & 0.821 & \\
\hline & & TT & 240 & 10.82 & 208 & 11.42 & & $0.934(0.759-1.150)$ & 0.522 & \\
\hline ITGB5 & rs6768588 & AA & 1139 & 51.19 & 939 & 51.79 & 0.9298 & 1.000 (reference) & & 0.719 \\
\hline & & AG & 919 & 41.30 & 740 & 40.82 & & $1.024(0.899-1.166)$ & 0.722 & \\
\hline & & GG & 167 & 7.51 & 134 & 7.39 & & $1.027(0.806-1.310)$ & 0.827 & \\
\hline & rs9824873 & TT & 1014 & 45.84 & 816 & 45.13 & 0.9025 & 1.000 (reference) & & 0.708 \\
\hline & & TC & 960 & 43.40 & 796 & 44.03 & & $0.971(0.851-1.107)$ & 0.656 & \\
\hline & & $\mathrm{CC}$ & 238 & 10.76 & 196 & 10.84 & & $0.977(0.792-1.206)$ & 0.830 & \\
\hline & rs6854472 & GG & 1884 & 83.92 & 1586 & 86.15 & 0.1370 & 1.000 (reference) & & 0.047 \\
\hline & & GT & 347 & 15.46 & 246 & 13.36 & & $1.187(0.995-1.417)$ & 0.056 & \\
\hline & & TT & 14 & 0.62 & 9 & 0.49 & & $1.310(0.565-3.033)$ & 0.529 & \\
\hline & rs12188136 & AA & 1050 & 47.36 & 917 & 50.16 & 0.0493 & 1.000 (reference) & & 0.367 \\
\hline & & AG & 981 & 44.25 & 740 & 40.48 & & 1.158 (1.016-1.319) & 0.027 & \\
\hline & & GG & 186 & 8.39 & 171 & 9.35 & & $0.950(0.758-1.191)$ & 0.656 & \\
\hline UNC5CL & rs2294693 & TT & 1268 & 56.78 & 1035 & 56.65 & 0.1972 & 1.000 (reference) & & 0.445 \\
\hline & & TC & 835 & 37.39 & 661 & 36.18 & & $1.031(0.905-1.175)$ & 0.647 & \\
\hline & & $\mathrm{CC}$ & 130 & 5.82 & 131 & 7.17 & & $0.810(0.627-1.047)$ & 0.107 & \\
\hline & rs9364414 & GG & 818 & 36.83 & 718 & 39.34 & 0.1831 & 1.000 (reference) & & 0.307 \\
\hline & & GA & 1086 & 48.90 & 841 & 46.08 & & 1.133 (0.991-1.297) & 0.068 & \\
\hline & & AA & 317 & 14.27 & 266 & 14.58 & & $1.046(0.864-1.267)$ & 0.645 & \\
\hline & rs7916519 & GG & 1306 & 58.62 & 1085 & 59.39 & 0.8840 & 1.000 (reference) & & 0.636 \\
\hline & & GA & 801 & 35.95 & 645 & 35.30 & & $1.032(0.905-1.176)$ & 0.641 & \\
\hline & & AA & 121 & 5.43 & 97 & 5.31 & & $1.036(0.784-1.370)$ & 0.802 & \\
\hline DYNC2H1 & rs11225815 & TT & 1265 & 56.73 & 999 & 55.32 & 0.6251 & 1.000 (reference) & & 0.488 \\
\hline & & $\mathrm{TC}$ & 824 & 36.95 & 694 & 38.43 & & $0.938(0.823-1.069)$ & 0.334 & \\
\hline & & $\mathrm{CC}$ & 141 & 6.32 & 113 & 6.26 & & $0.985(0.759-1.279)$ & 0.912 & \\
\hline & rs10895458 & AA & 1800 & 80.65 & 1450 & 79.02 & 0.0916 & 1.000 (reference) & & 0.094 \\
\hline & & $\mathrm{AC}$ & 413 & 18.50 & 357 & 19.46 & & $0.932(0.796-1.091)$ & 0.381 & \\
\hline & & $\mathrm{CC}$ & 19 & 0.85 & 28 & 1.53 & & $0.547(0.304-0.983)$ & 0.044 & \\
\hline OPCML & rs4578395 & TT & 1845 & 82.88 & 1501 & 82.43 & 0.9295 & 1.000 (reference) & & 0.705 \\
\hline & & $\mathrm{TC}$ & 368 & 16.53 & 309 & 16.97 & & $0.969(0.821-1.144)$ & 0.709 & \\
\hline & & $\mathrm{CC}$ & 13 & 0.58 & 11 & 0.60 & & $0.961(0.430-2.152)$ & 0.924 & \\
\hline & rs11059556 & $\mathrm{CC}$ & 961 & 43.29 & 799 & 44.14 & 0.6764 & 1.000 (reference) & & 0.863 \\
\hline & & $\mathrm{CT}$ & 1016 & 45.77 & 804 & 44.42 & & 1.051 (0.921-1.199) & 0.462 & \\
\hline & & TT & 243 & 10.95 & 207 & 11.44 & & $0.976(0.793-1.201)$ & 0.819 & \\
\hline & rs2025245 & GG & 907 & 40.55 & 708 & 38.75 & 0.2705 & 1.000 (reference) & & 0.119 \\
\hline & & GA & 1030 & 46.04 & 845 & 46.25 & & $0.951(0.832-1.088)$ & 0.467 & \\
\hline & & AA & 300 & 13.41 & 274 & 15.00 & & $0.855(0.706-1.035)$ & 0.107 & \\
\hline GPC5 & rs9584006 & $\mathrm{TT}$ & 780 & 35.15 & 609 & 33.65 & 0.3271 & 1.000 (reference) & & 0.154 \\
\hline & & TG & 1077 & 48.54 & 876 & 48.40 & & $0.960(0.836-1.103)$ & 0.563 & \\
\hline & & GG & 362 & 16.31 & 325 & 17.96 & & $0.870(0.724-1.045)$ & 0.136 & \\
\hline FMN1 & rs347940 & AA & 911 & 41.00 & 745 & 41.64 & 0.6201 & 1.000 (reference) & & 0.961 \\
\hline & & AG & 1037 & 46.67 & 810 & 45.28 & & 1.047 (0.916-1.196) & 0.500 & \\
\hline & & GG & 274 & 12.33 & 234 & 13.08 & & $0.958(0.784-1.169)$ & 0.670 & \\
\hline RORA & rs875339 & $\mathrm{CC}$ & 1104 & 49.44 & 881 & 48.35 & 0.0341 & 1.000 (reference) & & 0.091 \\
\hline & & $\mathrm{CT}$ & 939 & 42.05 & 742 & 40.72 & & $1.010(0.886-1.151)$ & 0.883 & \\
\hline & & TT & 190 & 8.51 & 199 & 10.92 & & $0.762(0.613-0.947)$ & 0.014 & \\
\hline
\end{tabular}




\begin{tabular}{|c|c|c|c|c|c|c|c|c|c|c|}
\hline \multirow[t]{2}{*}{ Gene } & \multirow[t]{2}{*}{ SNP ID } & \multirow[t]{2}{*}{ Genotype } & \multicolumn{2}{|l|}{ Case } & \multicolumn{2}{|c|}{ Control } & \multirow[t]{2}{*}{$P(2 d f)^{a}$} & \multicolumn{2}{|l|}{ Logistic regression } & \multirow[t]{2}{*}{$P_{\text {trend }}$} \\
\hline & & & No. & Frequency (\%) & No. & Frequency (\%) & & OR (95\%CI) & $\mathbf{P b}$ & \\
\hline \multirow[t]{3}{*}{ SNX29 } & rs12922317 & GG & 1090 & 49.05 & 841 & 46.08 & 0.1695 & 1.000 (reference) & & 0.091 \\
\hline & & GA & 929 & 41.81 & 808 & 44.27 & & $0.887(0.779-1.011)$ & 0.072 & \\
\hline & & AA & 203 & 9.14 & 176 & 9.64 & & $0.890(0.713-1.110)$ & 0.301 & \\
\hline
\end{tabular}

a. Global $P$ values [2 degrees of freedom (df)]: genotype frequencies in esophageal cancer and control group were compared using a $\chi^{2}$ test with 2 df. $\mathbf{b}$. P values from unconditional logistic regression analyses.

Table 4. Association analysis of 24 validated SNPs under dominant and recessive genetic model.

\begin{tabular}{|c|c|c|c|c|c|c|}
\hline \multirow[t]{2}{*}{ Gene } & \multirow[t]{2}{*}{ SNP ID } & \multirow[t]{2}{*}{ Genetic model } & \multirow[t]{2}{*}{ Case } & \multirow[t]{2}{*}{ Control } & \multicolumn{2}{|l|}{ Logistic regressiona $^{a}$} \\
\hline & & & & & OR $(95 \% \mathrm{CI})$ & Pc \\
\hline \multirow[t]{8}{*}{ SERINC2 } & rs4478858 & $(\mathrm{GA}+\mathrm{AA})$ vs. GG & $838 / 1380$ & $641 / 1185$ & $1.123(0.987-1.277)$ & 0.079 \\
\hline & & AA vs. $(\mathrm{GG}+\mathrm{GA})$ & $106 / 2112$ & $74 / 1752$ & $1.188(0.877-1.610)$ & 0.265 \\
\hline & rs10881372 & $(\mathrm{CT}+\mathrm{TT})$ vs. $\mathrm{CC}$ & $763 / 1481$ & $654 / 1172$ & $0.923(0.811-1.051)$ & 0.227 \\
\hline & & TT vs. $(\mathrm{CC}+\mathrm{CT})$ & $75 / 2169$ & $62 / 1764$ & $0.984(0.699-1.385)$ & 0.925 \\
\hline & rs10801638 & $(\mathrm{CT}+\mathrm{TT})$ vs. $\mathrm{CC}$ & $1139 / 1096$ & $934 / 891$ & $0.991(0.876-1.122)$ & 0.891 \\
\hline & & TT vs. $(\mathrm{CC}+\mathrm{CT})$ & $219 / 2016$ & $169 / 1656$ & $1.064(0.862-1.314)$ & 0.562 \\
\hline & rs10173378 & $(\mathrm{AG}+\mathrm{GG})$ vs. AA & $888 / 1341$ & $780 / 1053$ & $0.894(0.788-1.014)$ & 0.080 \\
\hline & & GG vs. $(A A+A G)$ & $99 / 2130$ & $102 / 1731$ & $0.789(0.594-1.048)$ & 0.101 \\
\hline \multirow[t]{2}{*}{ LYPD6 } & rs888103 & $(\mathrm{CT}+\mathrm{TT})$ vs. $\mathrm{CC}$ & $493 / 1743$ & $400 / 1432$ & $1.013(0.872-1.176)$ & 0.870 \\
\hline & & TT vs. $(\mathrm{CC}+\mathrm{CT})$ & $37 / 2199$ & $21 / 1811$ & $1.451(0.846-2.488)$ & 0.176 \\
\hline \multirow[t]{4}{*}{ BZW1 } & rs3815501 & $(\mathrm{GA}+\mathrm{AA})$ vs. GG & $1601 / 633$ & $1311 / 516$ & $0.995(0.868-1.142)$ & 0.948 \\
\hline & & AA vs. $(\mathrm{GG}+\mathrm{GA})$ & $486 / 1748$ & $386 / 1441$ & $1.038(0.893-1.207)$ & 0.628 \\
\hline & rs6717108 & $(\mathrm{CT}+\mathrm{TT})$ vs. $\mathrm{CC}$ & $1538 / 689$ & $1245 / 575$ & $1.031(0.902-1.178)$ & 0.655 \\
\hline & & TT vs. $(\mathrm{CC}+\mathrm{CT})$ & $463 / 1764$ & $370 / 1450$ & 1.029 (0.882-1.199) & 0.718 \\
\hline \multirow[t]{2}{*}{ UMPS } & rs10934685 & $(\mathrm{CT}+\mathrm{TT})$ vs. $\mathrm{CC}$ & $1246 / 972$ & $1035 / 787$ & $0.975(0.860-1.105)$ & 0.688 \\
\hline & & TT vs. $(\mathrm{CC}+\mathrm{CT})$ & $240 / 1978$ & $208 / 1614$ & $0.942(0.773-1.146)$ & 0.549 \\
\hline \multirow[t]{8}{*}{ ITGB5 } & rs6768588 & $(\mathrm{AG}+\mathrm{GG})$ vs. AA & $1086 / 1139$ & $874 / 939$ & $1.024(0.905-1.160)$ & 0.704 \\
\hline & & GG vs. $(A A+A G)$ & $167 / 2058$ & $134 / 1679$ & $1.017(0.803-1.288)$ & 0.890 \\
\hline & rs9824873 & $(\mathrm{TC}+\mathrm{CC})$ vs. TT & $1198 / 1014$ & $992 / 816$ & $0.972(0.858-1.101)$ & 0.654 \\
\hline & & CC vs. (TT+TC) & $238 / 1974$ & $196 / 1612$ & $0.992(0.812-1.211)$ & 0.934 \\
\hline & rs6854472 & $(\mathrm{GT}+\mathrm{TT})$ vs. GG & $361 / 1884$ & $255 / 1586$ & $1.192(1.002-1.418)$ & 0.048 \\
\hline & & TT vs. $(\mathrm{GG}+\mathrm{GT})$ & $14 / 2231$ & 9/1832 & $1.277(0.552-2.958)$ & 0.568 \\
\hline & rs12188136 & $(\mathrm{AG}+\mathrm{GG})$ vs. AA & $1167 / 1050$ & $911 / 917$ & $1.119(0.988-1.266)$ & 0.076 \\
\hline & & GG vs. $(A A+A G)$ & $186 / 2031$ & $171 / 1657$ & $0.887(0.714-1.103)$ & 0.282 \\
\hline \multirow[t]{6}{*}{ UNC5CL } & rs2294693 & $(\mathrm{TC}+\mathrm{CC})$ vs. TT & $965 / 1268$ & $792 / 1035$ & $0.995(0.878-1.127)$ & 0.932 \\
\hline & & CC vs. (TT+TC) & $130 / 2103$ & $131 / 1696$ & $0.800(0.623-1.029)$ & 0.082 \\
\hline & rs9364414 & $(\mathrm{GA}+\mathrm{AA})$ vs. GG & $1403 / 818$ & $1107 / 718$ & $1.112(0.979-1.264)$ & 0.101 \\
\hline & & AA vs. (GG+GA) & $317 / 1904$ & $266 / 1559$ & $0.976(0.818-1.164)$ & 0.785 \\
\hline & rs7916519 & $(\mathrm{GA}+\mathrm{AA})$ vs. $\mathrm{GG}$ & $922 / 1306$ & $742 / 1085$ & $1.032(0.910-1.171)$ & 0.620 \\
\hline & & AA vs. (GG+GA) & $121 / 2107$ & $97 / 1730$ & $1.024(0.778-1.348)$ & 0.864 \\
\hline \multirow[t]{4}{*}{ DYNC2H1 } & rs11225815 & $(\mathrm{TC}+\mathrm{CC})$ vs. TT & $965 / 1265$ & $807 / 999$ & $0.944(0.833-1.070)$ & 0.369 \\
\hline & & CC vs. $(\mathrm{TT}+\mathrm{TC})$ & $141 / 2089$ & $113 / 1693$ & $1.011(0.783-1.306)$ & 0.932 \\
\hline & rs10895458 & $(\mathrm{AC}+\mathrm{CC})$ vs. $\mathrm{AA}$ & $432 / 1800$ & $385 / 1450$ & $0.904(0.775-1.054)$ & 0.198 \\
\hline & & $\mathrm{CC}$ vs. $(\mathrm{AA}+\mathrm{AC})$ & $19 / 2213$ & $28 / 1807$ & $0.554(0.308-0.995)$ & 0.048 \\
\hline \multirow[t]{6}{*}{ OPCML } & rs4578395 & $(\mathrm{TC}+\mathrm{CC})$ vs. TT & $381 / 1845$ & $320 / 1501$ & $0.969(0.823-1.141)$ & 0.702 \\
\hline & & CC vs. $(\mathrm{TT}+\mathrm{TC})$ & $13 / 2213$ & $11 / 1810$ & $0.967(0.432-2.163)$ & 0.934 \\
\hline & rs11059556 & $(\mathrm{CT}+\mathrm{TT})$ vs. $\mathrm{CC}$ & $1259 / 961$ & $1011 / 799$ & $1.035(0.914-1.173)$ & 0.586 \\
\hline & & TT vs. $(\mathrm{CC}+\mathrm{CT})$ & $243 / 1977$ & $207 / 1603$ & $0.952(0.782-1.159)$ & 0.623 \\
\hline & rs2025245 & $(\mathrm{GA}+\mathrm{AA})$ vs. GG & $1330 / 907$ & $1119 / 708$ & $0.928(0.818-1.053)$ & 0.245 \\
\hline & & AA vs. $(\mathrm{GG}+\mathrm{GA})$ & $300 / 1937$ & $274 / 1553$ & $0.878(0.736-1.048)$ & 0.149 \\
\hline \multirow[t]{2}{*}{ GPC5 } & rs9584006 & (TG+GG) vs. TT & $1439 / 780$ & $1201 / 609$ & $0.935(0.821-1.066)$ & 0.318 \\
\hline & & GG vs. $(\mathrm{TT}+\mathrm{TG})$ & $362 / 1857$ & $325 / 1485$ & $0.891(0.756-1.050)$ & 0.168 \\
\hline \multirow[t]{2}{*}{ FMN1 } & rs 347940 & $(\mathrm{AG}+\mathrm{GG})$ vs. AA & $1311 / 911$ & $1044 / 745$ & $1.027(0.905-1.165)$ & 0.680 \\
\hline & & GG vs. $(A A+A G)$ & $274 / 1948$ & $234 / 1555$ & $0.935(0.775-1.127)$ & 0.479 \\
\hline \multirow[t]{2}{*}{ RORA } & rs 875339 & $(\mathrm{CT}+\mathrm{TT})$ vs. $\mathrm{CC}$ & $1129 / 1104$ & $941 / 881$ & $0.957(0.846-1.084)$ & 0.491 \\
\hline & & TT vs. $(\mathrm{CC}+\mathrm{CT})$ & $190 / 2043$ & $199 / 1623$ & $0.758(0.615-0.935)$ & 0.010 \\
\hline SNX29 & rs12922317 & $(\mathrm{GA}+\mathrm{AA})$ vs. GG & $1132 / 1090$ & $984 / 841$ & $0.888(0.784-1.005)$ & 0.060 \\
\hline SERINC2 & & AA vs. (GG+GA) & $203 / 2019$ & $176 / 1649$ & $0.942(0.762-1.165)$ & 0.581 \\
\hline
\end{tabular}

a. $P$ values from unconditional logistic regression analyses.

The present study is not without limitations. Statistical correction was used to adjust for multiple testing for a specific gene, but this is controversial. The Bonferroni correction and Bayesian techniques are frequently used, but they are problematic when correcting multiple comparisons [41] and such corrections might not be needed when different associations are of interest on a purely one-at-a-time basis $[42,43]$. Secondly, our study included patients with first ESCC and second ESCC. First ESCC is more relevant to genetic factors than second ESCC. Thirdly, although our study suggested that some loci may be involved in the prevalence of acquired ESCC, only selected SNPs based on the literature were examined and they might not be enough to describe the entire genetic variation of Han Chinese. Finally, this was a 
retrospective study and data about lifestyle habits (especially smoking and drinking) were not available or reliable for all patients, preventing subgroup and interaction analyses. Beyond the association studies, the literature is currently limited by the lack of mechanistic studies about the involvement of these SNPs in the development of ESCC and the present study was not designed to determine those mechanisms. Additional studies will have to be carried out on this issue.

\section{Conclusion}

This large-scale replication study showed that rs12188136 and rs6854472 are associated with ESCC in a Han Chinese subgroup from Eastern China, and that rs875339 is negative associated with ESCC. This study underlines the genetic complexity of ESCC development.

\section{Acknowledgements}

This study was supported by The National Health and Family Planning Commission Scientific Research Foundation-Zhejiang Medical and Health Major Science and Technology Plan (WKJ-ZJ-1505), National Natural Science Foundation of China (U1604189), the Leading Talents in Scientific and Technological Innovation from Zhejiang Provincial Ten Thousand Talents Plan (Zhejiang Provincial CPC Committee Talents [2019]-3), Zhejiang Provincial Program for the Cultivation of High-Level Innovative Health Talents (Zjwjw2014-108), and the Major Training Personnel from Zhejiang Provincial Program for the Training and Development Project for 151 Talents (Zjhrss2014-150). The authors acknowledge the help of Yu Zhong and Qing-Wei Ma from Beijing YiXin BoChuang Biotechnology Co., Ltd (5 Floor, Boda Building, Zhongguancun Life Science Park, Changping District, Beijing 102206, China) for technical support in SNP genotyping assays.

\section{Author contributions}

Wang KL, Chen XL, Lei L, Li P and Ling ZQ contributed to the design, execution, and analysis of this paper. Wang KL, Chen XL, Lei L, Li P and Ling ZQ drafted the manuscript. Hong LL and Huang XC provided some help for data analysis. All the authors (including Mao WM) were involved in the critical revision of the manuscript.

\section{Ethics statement}

The study was performed in accordance with the Declaration of Helsinki and approved by the Ethics Committees of Zhejiang Cancer Hospital. Written informed consent was obtained for the recruitment of each participant. Each participant was then interviewed to collect detailed information on demographic characteristics.

\section{Competing Interests}

The authors have declared that no competing interest exists.

\section{References}

1. Gupta B, Kumar N. Worldwide incidence, mortality and time trends for cancer of the oesophagus. Eur J Cancer Prev. 2017; 26:107-118.

2. Ferlay J, Shin HR, Bray F, et al. Estimates of worldwide burden of cancer in 2008: GLOBOCAN 2008. Int J Cancer. 2010; 127:2893-917.

3. Abnet CC, Arnold M, Wei WQ. Epidemiology of Esophageal Squamous Cell Carcinoma. Gastroenterology. 2018; 154:360-373.

4. Zeng H, Chen W, Zheng R, et al. Changing cancer survival in China during 2003-15: a pooled analysis of 17 population-based cancer registries. Lancet Glob Health. 2018; 6:e555-e567.

5. Chen W, Sun K, Zheng R, et al. Cancer incidence and mortality in China, 2014. Chin J Cancer Res. 2018; 30:1-12.

6. Zhou L, Fu G, Wei J, et al. The identification of two regulatory ESCC susceptibility genetic variants in the TERT-CLPTM1L loci. Oncotarget. 2016; 7: 5495-506.

7. Li K, Yin X, Yang H, et al. Association of the genetic polymorphisms in XRCC6 and XRCC5 with the risk of ESCC in a high-incidence region of North China. Tumori. 2015; 101:24-9.

8. Zhang H, Li H, Yu H. Analysis of the role of rs2031920 and rs3813867 polymorphisms within the cytochrome P450 2E1 gene in the risk of squamous cell carcinoma. Cancer Cell Int. 2018; 18:67.

9. Moghadam AR, Mehramiz M, Entezari M, et al. A genetic polymorphism in the CYP1B1 gene in patients with squamous cell carcinoma of the esophagus: an Iranian Mashhad cohort study recruited over 10 years. Pharmacogenomics. 2018; 19:539-546.

10. Yin J, Tang W, Long T, et al. Association of ALDH3B2 gene polymorphism and risk factors with susceptibility of esophageal squamous cell carcinoma in a Chinese population: a case-control study involving 2,358 subjects. Oncotarget. 2017; 8: 110153-110165.

11. Chen $\mathrm{T}$, Cheng $\mathrm{H}, \mathrm{Chen} \mathrm{X}$, et al. Family history of esophageal cancer increases the risk of esophageal squamous cell carcinoma. Sci Rep. 2015; 5:16038.

12. Bhat GA, Shah IA, Rafiq R, et al. Family history of cancer and the risk of squamous cell carcinoma of oesophagus: a case-control study in Kashmir, India. Br J Cancer. 2015; 113:524-32.

13. Jia N, Wen $\mathrm{X}$, Zhang $\mathrm{N}$, et al. Younger age of onset and multiple primary lesions associated with esophageal squamous cell carcinoma cases with a positive family history of the cancer suggests genetic predisposition. Chin Med J (Engl). 2014; 127: 2779-83.

14. Chang J, Huang Y, Wei L, et al. Risk prediction of esophageal squamous-cell carcinoma with common genetic variants and lifestyle factors in Chinese population. Carcinogenesis 2013;34:1782-6.

15. Jessri M, Rashidkhani B, Hajizadeh B, et al. Adherence to Mediterranean-style dietary pattern and risk of esophageal squamous cell carcinoma: a case-control study in Iran. J Am Coll Nutr. 2012; 31:338-51.

16. Wu IC, Wu CC, Lu CY, et al. Substance use (alcohol, areca nut and cigarette) is associated with poor prognosis of esophageal squamous cell carcinoma. PloS one 2013; 8:e55834.

17. Taccioli $\mathrm{C}$, Chen $\mathrm{H}$, Jiang $\mathrm{Y}$, et al. Dietary zinc deficiency fuels esophageal cancer development by inducing a distinct inflammatory signature. Oncogene 2012; 31:4550-8.

18. Guo W, Zhao YP, Jiang YG, et al. Restoring the metabolic disturbance of zinc: may not only contribute to the prevention of esophageal squamous cell cancer. Med Hypotheses. 2008; 71:957-9.

19. Tan W, Miao X, Wang L, et al. Significant increase in risk of gastroesophageal cancer is associated with interaction between promoter polymorphisms in thymidylate synthase and serum folate status. Carcinogenesis. 2005; 26:1430-5.

20. Stolzenberg-Solomon RZ, Qiao YL, Abnet CC, et al. Esophageal and gastric cardia cancer risk and folate- and vitamin $\mathrm{B}(12)$-related polymorphisms in Linxian, China. Cancer Epidemiol Biomarkers Prev. 2003; 12:1222-6.

21. Oka M, Yamamoto K, Takahashi M, et al. Relationship between serum levels of interleukin 6, various disease parameters and malnutrition in patients with esophageal squamous cell carcinoma. Cancer Res. 1996; 56:2776-80.

22. Chen $\mathrm{CH}$, Lu HI, Wang YM, et al. Areca nut is associated with younger age of diagnosis, poor chemoradiotherapy response, and shorter overall survival in esophageal squamous cell carcinoma. PloS one. 2017; 12:e0172752.

23. De Stefani E, Deneo-Pellegrini H, Ronco AL, et al. Meat consumption, cooking methods, mutagens, and risk of squamous cell carcinoma of the esophagus: a case-control study in Uruguay. Nutr Cancer. 2012; 64:294-9.

24. Cao F, Han H, Zhang F, et al. HPV infection in esophageal squamous cell carcinoma and its relationship to the prognosis of patients in northern China. ScientificWorldJournal. 2014; 2014:804738. 
25. Yang J, Liu B, Li W, et al. Association of $\mathrm{p} 53$ and MDM2 polymorphisms with risk of human papillomavirus (HPV)-related esophageal squamous cell carcinoma (ESCC). Cancer Epidemiol. 2013; 37:629-33.

26. Yang J, Wu H, Wei S, et al. HPV seropositivity joints with susceptibility loci identified in GWASs at apoptosis associated genes to increase the risk of Esophageal Squamous Cell Carcinoma (ESCC). BMC cancer. 2014; 14:501.

27. Tang WR, Chen ZJ, Lin K, et al. Development of esophageal cancer in Chaoshan region, China: association with environmental, genetic and cultural factors. Int J Hyg Environ Health. 2015; 218:12-8.

28. Chen J, Kwong DL, Cao T, et al. Esophageal squamous cell carcinoma (ESCC): advance in genomics and molecular genetics. Dis Esophagus. 2015; 28:84-9.

29. Gholipour M, Islami F, Roshandel G, et al. Esophageal Cancer in Golestan Province, Iran: A Review of Genetic Susceptibility and Environmental Risk Factors. Middle East J Dig Dis. 2016; 8:249-266.

30. Hu N, Wang C, Hu Y, et al. Genome-wide association study in esophageal cancer using GeneChip mapping 10K array. Cancer Res. 2005; 65:2542-6.

31. Abnet CC, Freedman ND, Hu N, et al. A shared susceptibility locus in PLCE1 at $10 \mathrm{q} 23$ for gastric adenocarcinoma and esophageal squamous cell carcinoma. Nat Genet. 2010; 42:764-7.

32. Wang LD, Zhou FY, Li XM, et al. Genome-wide association study of esophageal squamous cell carcinoma in Chinese subjects identifies susceptibility loci at PLCE1 and C20orf54. Nat Genet. 2010; 42:759-63.

33. Wu C, Hu Z, He Z, et al. Genome-wide association study identifies three new susceptibility loci for esophageal squamous-cell carcinoma in Chinese populations. Nat Genet. 2011; 43:679-84.

34. Wu C, Kraft P, Zhai K, et al. Genome-wide association analyses of esophageal squamous cell carcinoma in Chinese identify multiple susceptibility loci and gene-environment interactions. Nat Genet. 2012; 44:1090-7.

35. Wu C, Li D, Jia W, et al. Genome-wide association study identifies common variants in SLC39A6 associated with length of survival in esophageal squamous-cell carcinoma. Nat Genet. 2013; 45:632-8.

36. Lordick F, Mariette C, Haustermans K, et al. Oesophageal cancer: ESMO Clinical Practice Guidelines for diagnosis, treatment and follow-up. Ann Oncol. 2016; 27(suppl 5):v50-v57.

37. Zhao LQ, Wen ZJ, Wei $Y$, et al. Polymorphisms of renin-angiotensin-aldosterone system gene in chinese han patients with nonfamilial atrial fibrillation. PloS one 2015; 10:e0117489.

38. Akaike H. A new look at the statistical model identification. IEEE Transactions on Automatic Control. 1974; 19:716-23.

39. $\mathrm{Hu} \mathrm{H}$, Yang J, Sun Y, et al. Putatively functional PLCE1 variants and susceptibility to esophageal squamous cell carcinoma (ESCC): a case-control study in eastern Chinese populations. Ann Surg Oncol. 2012; 19:2403-10.

40. Wang J, Wei J, Xu X, et al. Replication study of ESCC susceptibility genetic polymorphisms locating in the ADH1B-ADH1C-ADH7 cluster identified by GWAS. PloS one. 2014; 9:e94096.

41. Benjamini $Y$, Hochberg Y. Controlling the false discovery rate: A practical and powerful approach to multiple testing. J R Statist Soc B. 1995; 57:289-300.

42. Perneger TV. What's wrong with Bonferroni adjustments. Bmj. 1998; 316:12368.

43. Rothman KJ. No adjustments are needed for multiple comparisons. Epidemiology. 1990; 1:43-6. 